\title{
Morphological Diversity of Some Tephrosia Species (Fabaceae) in Saudi Arabia
}

\author{
Faraj A. Al-Ghamdi \\ Department of Biological Science, Faculty of Science, Jeddah, KSA. \\ Email: faalghamdi@kau.edu.sa \\ Received January $6^{\text {th }}$, 2013; revised February $10^{\text {th }}$, 2013; accepted February $27^{\text {th }}, 2013$
}

\begin{abstract}
Morphological features of nine wild species belonging to the genus Tephrosia PERS growing in Saudi Arabia were extensively studied in order to support the diversity and simplify the identification of these species. The studied features included macro and micro morphological features of stem, leaves, flowers and pods. The most important features regarded for distinction of Saudi Arabia species of Tephrosia were pods shape, color, size, dehiscence and number of seeds per pod, inflorescences position, flower opening, shape of the upper 2 teeth of calyx, shape of sepals apex, shape of wings, length of hairs on ovary, direction of staminal tube tip, leaflets shape and surface and stem habit form and life spin. A key for the identification of the investigated species based on these morphological features is provided.
\end{abstract}

Keywords: Morphology; Tephrosia; Fabaceae; Saudi Arabia

\section{Introduction}

The genus Tephrosia PERS. (Fabaceae, Papilionoideae) comprises between 300 to 400 species of annual and perennial woody herb, distributed in tropical and subtropical regions of the world [1,2].

In Saudi Arabia, there are about 11 taxa of Tephrosia, which are mainly distributed in the northwestern, western, south and southwestern regions [3-7]. Plant morphology has served largely for systematics, using morphological characteristics to carve up diversity into its systematic subunits [8].

Eight species of Tephrosia growing in Saudi Arabia were described by [7], namely $T$. desertorum, $T$. heterophylla (T. schweinfurthii), T. nubica, T. pumila, T. purpurea, T. subtriflora, T. uniflora and T. villosa. But specimens of $T$. villosa described as a species of Saudi Arabia flora [7], whereas plant specimen was collected from Ras al-Khaimah, United Arab of Emirates. Eleven species of Tephrosia were described briefly [5] including, T. apollina, T. leptostachya and T. pubescens which treated as subspecies or varieties of T. purpurea, in addition to T. quartiniana and other species recorded by [7] except $T$. villosa.

Tephrosia schweinfurthii and T. subtriflora, which are characterized by their 1 -foliolate leaves, were recorded as rare and endangered species [5].

The aim of this work is to revise systematically most of the native taxa of Tephrosia in Saudi Arabia with reference to the earlier applied characters as well as macro and micromorphological characters, which hope to be more reliable the diversity of these taxa.

\section{Material and Methods}

Morphological features of the studied species are based on the fresh plant samples collected from the sites which were reported by [4-7]; with field survey of the species throughout Saudi Arabia regions in addition to available herbarium specimens (Table 1). The plant specimens nomenclatured according to [5-7,9].

Field studies included growth characters, habit of stem, plant height, flower opening and corolla color, Microscopic characters were obtained by using binocular microscope (Zeiss Stemi 2000-Germany). Herbarium specimens of the studied species were prepared and deposited in the Herbarium of the Biology Department Faculty of Science, King Abdulaziz University Terminology used here follows [10].

\section{Results and Discussion}

The observed morphological characters are listed in Tables 2-5. The most important characters are discussed below.

\subsection{Vegetative Characters}

The stems of studied Tephrosia species are erect, ascending or prostrate. The perennial suffrutescent (herb woody at the base) are observed in T. appolinea, T. pu- 
Table 1. List and sites of collection of the studied Tephrosia species with relevant information about their herbarium samples.

\begin{tabular}{|c|c|}
\hline Taxa & Location/Collector \\
\hline $\begin{array}{l}\text { T. purpurea (L.) PERS. subsp. } \\
\text { appolinea (DELILE) HOSNI \& } \\
\text { EL-KAREMY. }\end{array}$ & $\begin{array}{l}\text { Wadi Assafraa, Bader, } 100 \mathrm{~km} \\
\text { South of Almadinah, Al-Zahrani R. } \\
\text { (R 1079). }\end{array}$ \\
\hline T. desertorum SCHEELE. & $\begin{array}{l}\text { East Jeddah, Al-Zahrani R. } \\
\text { (R 1478). }\end{array}$ \\
\hline T. nubica (BOISS.) BAKER. & $\begin{array}{l}\text { Umm Hablain, Jeddah, Al-Zahrani } \\
\text { R. (R 1980). }\end{array}$ \\
\hline $\begin{array}{l}\text { T. purpurea (L.) PERS. subsp. } \\
\text { leptostachya (DC.) BRUMMITT } \\
\text { var. pubescens BAKER. }\end{array}$ & $\begin{array}{l}\text { Wadi Noaman, Makkah Taif } \\
\text { (Al-Hada) Road, Al-Zahrani R. } \\
\text { (R 1861). }\end{array}$ \\
\hline $\begin{array}{l}\text { T. purpurea (L.) PERS. subsp. } \\
\text { leptostachya (DC.) BRUMMITT } \\
\text { var. leptostachya }\end{array}$ & $\begin{array}{l}\text { East Jeddah, Al-Zahrani R. } \\
\text { (R 1183). }\end{array}$ \\
\hline T. pumila (LAM.) PERS. & $\begin{array}{l}\text { Jabal Fafa, Elkhashah, East of } \\
\text { Jizan, Al-Zahrani R. (R 1982). }\end{array}$ \\
\hline T. purpurea (L.) PERS. & Misk Lake, Jeddah \\
\hline $\begin{array}{l}\text { T. quartiniana CUFOD. Ex } \\
\text { GREUTER \& BURDET }\end{array}$ & $\begin{array}{l}\text { East Jeddah, Al-Zahrani R. } \\
\text { (R 1284). }\end{array}$ \\
\hline T. uniflora PERS & $\begin{array}{l}\text { Bulghazi, Jizan, Al-Zahrani R. } \\
\text { (R 1105). }\end{array}$ \\
\hline
\end{tabular}

bescens, T. pumila, T. purpurea, T. quartiniana and T. uniflora, whereas annual suffrutescent in $T$. desertorum and T. leptostachya, and perennial shrubs in and T. nubica. The rough surface characterized stems of $T$. pubescens and T. pumila, while the surface of other studied species are smooth (Figure 1).

Reference [11] regarded the characters of leaf and flower grouping as the main features for the distinction of the Egyptian Tephrosia species. Leaves of the studied Tephrosia species are imparipinnate, petiolate, with narrowly triangular stipules. Leaflets are many 3 - 15 (number of leaflets per leaf are not stable for the same species), petiolate with petiole length range from 0.75 to $1.75 \mathrm{~mm}$. The leaflets arrangement is opposite without stipels. Leaflets are obovate, oblanceolate, or elliptic to oblanceolate, with entire margin. Leaflets apex was apiculate, emarginate or obcordate with acuminate tip (Table 2).

\subsection{Flower Characters}

Floral characters are the most important features that used in systematic of Papilionoideae [12]. Inflorescences of the studied Tephrosia species are Pseudoracemes, bracteolate (triangular $0.35-1 \times 1.25-3$ - $5 \mathrm{~mm}$ size), flowers with triangular bracteale. According to Pseudoracemes characters, studied Tephrosia species can be classified into three groups: the first includes $T$. appolinea, T. desertorum, T. leptostachya, T. nubica, T. pubescens, and T. pumila which characterized by inflorescences borne terminal (opposite the leaf). The second includes T. quartiniana and T. uniflora characterized by axilary inflorescences. Whereas T. purpurea was recognized in the third group by the inflorescences borne terminal (opposite to a leaf) or axillary. Based on the peduncle length, the first and third groups are padunculate ranged from 0 to $7 \mathrm{~cm}$ long, while the second group shows reduced peduncle. Others floral characters showed significant importance in distinction between the studied Tephrosia species are, Flower opening (day or evening opening), the shapes the 2 upper teeth of calyx (cleft or dentate), shape of sepals apex (acute or caedate), shape of wings (club, boat or kidney) length of hairs on ovary and direction of staminal tube tip (Figures 2-4).

\subsection{Fruits Characters}

Pods shapes and characters (color, size, dehiscence and number of seeds) are valuable for species identification [13]. With the exception of $T$. nubica, the other species of Tephrosia have linear, yellow to brown, many seeded (5 - 13) pods. Pods size range $0.25-0.4 \times 2.75-5.3 \mathrm{~cm}$, covered with short to medium white hairs, dehiscing at maturity. In T. nubica Pods are ovoid, one seeded, with

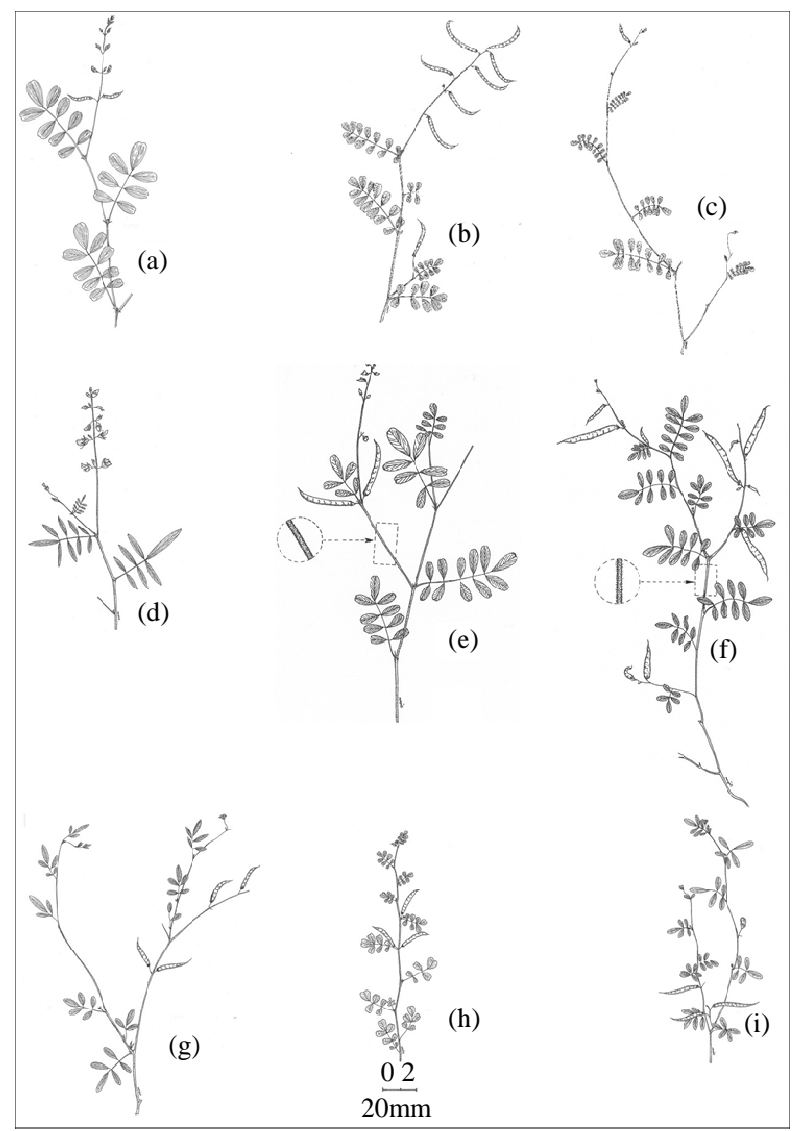

Figure 1. Schematic diagram showing arial parts of different Tephrosia species: (a) T. apollinea; (b) T. desertorum; (c) T. leptostachya; (d) T. nubica; (e) T. pubescens; (f) T. pumila; (g) T. purpurea; (h) T. quartiniana; (i) T. uniflora. 
Table 2. Vegetative characters of the studied Tephrosia species.

\begin{tabular}{|c|c|c|c|c|c|c|c|c|c|c|c|c|}
\hline \multirow[b]{2}{*}{ Taxa } & \multicolumn{3}{|c|}{ Stem } & \multirow{2}{*}{$\begin{array}{l}\text { Plant } \\
\text { length } \\
\text { (M) }\end{array}$} & \multicolumn{7}{|c|}{ Leaflets } & \multirow{2}{*}{$\begin{array}{l}\text { Stipule } \\
\text { size } \\
\text { mm }\end{array}$} \\
\hline & Habit & Life form & Surface & & shape & Size $\mathbf{c m}$ & numbers & apex & $\begin{array}{c}\text { Tip } \\
\text { length }\end{array}$ & $\begin{array}{c}\text { Petiole } \\
\text { length } \\
\text { Mm }\end{array}$ & Surface & \\
\hline T. apollinea & $\begin{array}{c}\text { Perennial } \\
\text { suffrutescent }\end{array}$ & Erect & smooth & 0.4 & Obovate & $\begin{array}{c}1.3-0.5 \times \\
3-1\end{array}$ & $9-5-(3)$ & \multicolumn{2}{|c|}{ Emarginate 0.25 mm } & 1.7 & pannose & $1 \times 8$ \\
\hline T. desertorum & $\begin{array}{c}\text { Annual } \\
\text { suffrutescent }\end{array}$ & Ascending & smooth & 0.4 & Obovate & $\begin{array}{c}1.3-0.6 \times \\
2.2-0.9\end{array}$ & $\begin{array}{c}13-9- \\
(7)-(15)\end{array}$ & Obcordate & 0.4 & 1.5 & pannose & $1 \times 5$ \\
\hline T. leptostachya & Annual herb & prostrate & smooth & 0.5 & Obovate & $\begin{array}{c}0.8-0.4 \times \\
1.5-0.4\end{array}$ & $17-9-(7)$ & Obcordate & 0.25 & 1 & pannose & $1 \times 4$ \\
\hline T. nubica & $\begin{array}{l}\text { Perennial } \\
\text { shrub }\end{array}$ & Erect & smooth & 1.5 & Oblanceolate & $\begin{array}{c}0.7-0.3 \times \\
4-1\end{array}$ & (9) - 7- 5 & Apiculate & 0.5 & 1.5 & pannose & $1.5 \times 3$ \\
\hline T. pubescens & $\begin{array}{c}\text { Perennial } \\
\text { suffrutescent }\end{array}$ & Ascending & rough & 0.4 & Oblanceolate & $\begin{array}{c}0.7-0.3 \times \\
2.2-0.6\end{array}$ & $9-(7-5)-13$ & Emarginate & 0.35 & 1. 7 & pubescent & $1 \times 4$ \\
\hline T. purpurea & $\begin{array}{c}\text { Perennial } \\
\text { shrub or } \\
\text { suffrutescent }\end{array}$ & $\begin{array}{c}\text { Erect/Asc } \\
\text { ending }\end{array}$ & smooth & 0.7 & $\begin{array}{c}\text { Elliptic/ } \\
\text { Oblanceolate }\end{array}$ & $\begin{array}{c}0.7-0.3 \times \\
2.6-0.9\end{array}$ & $5-3$ & Apiculate & 0.5 & 1 & pannose & $1 \times 6$ \\
\hline T. quartiniana & $\begin{array}{c}\text { Perennial } \\
\text { suffrutescent }\end{array}$ & prostrate & smooth & 0.7 & Obovate & $\begin{array}{c}1.1-0.3 \times \\
1.3-0.5\end{array}$ & (9) - 7 - 5 & Emarginate & 0.25 & 1 & $\begin{array}{l}\text { Pannose } \\
\text { down }\end{array}$ & $1 \times 3$ \\
\hline T. uniflora & $\begin{array}{c}\text { Perennial } \\
\text { suffrutescent }\end{array}$ & Erect & smooth & 0.6 & Oblanceolate & $\begin{array}{c}0.4-2 \times \\
0.03-0.7\end{array}$ & $7-5-(3)$ & Apiculate & 0.25 & 1 & pannose & $\begin{array}{c}0.75 \times \\
2.75\end{array}$ \\
\hline
\end{tabular}

All measures are given as a mean.

Table 3. Inflorescence and floral (calyx) characters of the studied Tephrosia species.

\begin{tabular}{|c|c|c|c|c|c|c|c|c|c|c|c|c|c|c|c|c|c|c|}
\hline \multirow[b]{4}{*}{ Taxa } & \multicolumn{3}{|c|}{ Inflorescence } & \multirow{4}{*}{ 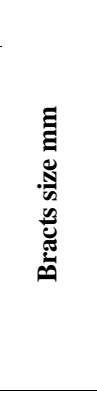 } & \multirow{4}{*}{ 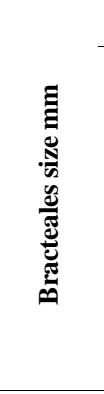 } & \multicolumn{13}{|c|}{ Flower } \\
\hline & \multirow[b]{3}{*}{ : } & \multirow[b]{3}{*}{ 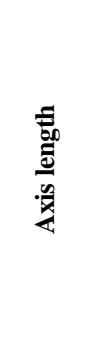 } & \multirow[b]{3}{*}{ 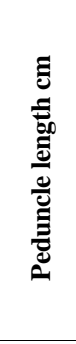 } & & & \multirow[b]{3}{*}{$\frac{n}{\frac{n}{2}}$} & \multirow[b]{3}{*}{ E } & \multirow[b]{3}{*}{ ڤ̆ } & \multirow[b]{3}{*}{ 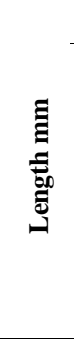 } & \multirow{3}{*}{\multicolumn{3}{|c|}{ 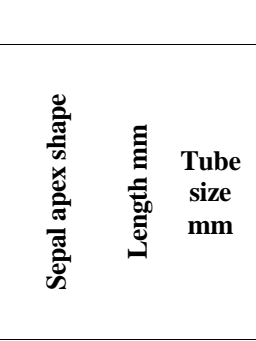 }} & \multicolumn{3}{|c|}{ Calyx } & \multirow{2}{*}{\multicolumn{2}{|c|}{ Lower teeth }} & \multirow[b]{3}{*}{ E } \\
\hline & & & & & & & & & & & & & Upper & r two te & teeth & & & \\
\hline & & & & & & & & & & & & & 䓂 & 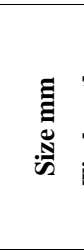 & 志 & 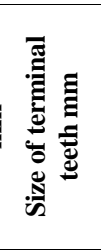 & 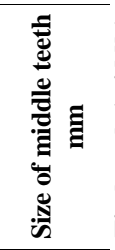 & \\
\hline T. apollinea & $\begin{array}{l}\text { Opposite } \\
\text { to leaf }\end{array}$ & $20-10$ & $6.5-0$ & $\begin{array}{c}0.5 \times \\
2.75\end{array}$ & $\begin{array}{c}0.25 \times \\
1.2\end{array}$ & $2-4$ & 2.75 & Day & 11.5 & Acute & 3.5 & $\begin{array}{c}2.25- \\
1.25\end{array}$ & Cleft & $\begin{array}{c}1.25 \times \\
3.25\end{array}$ & 1 & $\begin{array}{c}1 \times \\
3.255\end{array}$ & $1 \times 3.5$ & 2.25 \\
\hline T. desertorum & $\begin{array}{c}\text { Opposite } \\
\text { to leaf }\end{array}$ & $4-16$ & $5.8-0$ & $\begin{array}{c}0.5 \times \\
2.5\end{array}$ & $\begin{array}{c}0.25 \times \\
1.75\end{array}$ & $2-3$ & 2 & Day & 9.5 & Acute & 3.75 & $2-1.5$ & Cleft & $\begin{array}{c}1.5 \times \\
3\end{array}$ & 1.5 & $\begin{array}{l}1.5 \times \\
3.25\end{array}$ & $\begin{array}{l}1.5 \times \\
3.75\end{array}$ & 2.5 \\
\hline T. nubica & $\begin{array}{l}\text { Opposite } \\
\text { to leaf }\end{array}$ & $30-20$ & $7-0$ & $1 \times 3.5$ & $0.5 \times 2$ & $1-4$ & 5.5 & Evening & 13 & Acute & 7.5 & $2-2.25$ & Dentate & e $2 \times 5$ & 3 & $2 \times 6.5$ & $1.5 \times 7.5$ & 5 \\
\hline T. pubescens & $\begin{array}{l}\text { Opposite } \\
\text { to leaf }\end{array}$ & $14-5$ & $6.5-0$ & $\begin{array}{c}0.5 \times \\
2.5\end{array}$ & $\begin{array}{c}0.2 \times \\
0.8\end{array}$ & $1-3$ & 2.5 & Day & 10.25 & Acute & 3.5 & $\begin{array}{c}2.25- \\
1.25\end{array}$ & Cleft & $\begin{array}{c}1.25 \times \\
3.25\end{array}$ & 1 & $\begin{array}{c}1 \times \\
3.255\end{array}$ & $1 \times 3.5$ & 2.25 \\
\hline T. pumila & $\begin{array}{l}\text { Opposite } \\
\text { to leaf }\end{array}$ & $10-2$ & $1.5-0$ & $\begin{array}{c}0.35 \times 1 \\
25\end{array}$ & $\begin{array}{l}0.2 \times \\
0.75\end{array}$ & $1-2$ & 2 & Evening & 7 & Caedate & 2.75 & $1.25-1$ & Cleft & $1 \times 2$ & 1 & $1 \times 2.25$ & $1 \times 2.75$ & 1.75 \\
\hline T. purpurea & $\begin{array}{c}\text { Axillary or } \\
\text { opposite to } \\
\text { leaf }\end{array}$ & $15-0$ & $5-0$ & $2 \times 0.5$ & $0.25 \times 1$ & $2-5$ & 3 & Evening & 10.5 & Mucronte & e 3.25 & $2-1.5$ & Cleft & $\begin{array}{c}1.25 \times \\
3\end{array}$ & 1 & $1.25 \times 3$ & $\begin{array}{c}1.25 \times \\
3.25\end{array}$ & 1.75 \\
\hline T. quartiniana & Axillary & - & - & $\begin{array}{l}0.5 \times \\
2.75\end{array}$ & $0.25 \times 1$ & $1-3$ & 2 & Evening & 7 & Acute & 2.5 & $1.5-1$ & Cleft & $1 \times 1$ & 0.75 & $1 \times 2.5$ & $1 \times 2.5$ & 1.5 \\
\hline T. uniflora & Axillary & - & - & $2 \times 0.5$ & $\begin{array}{c}0.2 \times \\
1.25\end{array}$ & $1-2$ & 2.5 & Evening & 8.25 & Acute & 4.25 & $\begin{array}{c}2.25- \\
1.25\end{array}$ & Cleft & $1 \times 4$ & 1.75 & $1.25 \times 4$ & $1 \times 4.25$ & 3 \\
\hline
\end{tabular}

All measures are given as a mean. 
Table 4. Corolla and Androecium characters of the studied Tephrosia species.

\begin{tabular}{|c|c|c|c|c|c|c|c|c|c|c|c|c|}
\hline \multirow[b]{2}{*}{ Species } & \multirow[b]{2}{*}{ Color } & \multicolumn{4}{|c|}{ Corolla } & \multirow[b]{2}{*}{$\begin{array}{l}\text { Wings } \\
\text { shape }\end{array}$} & \multicolumn{5}{|c|}{ Androecium } & \multirow[b]{2}{*}{$\begin{array}{c}\text { Tip } \\
\text { direction }\end{array}$} \\
\hline & & $\begin{array}{c}\text { Corolla } \\
\text { length } \\
\text { mm }\end{array}$ & $\begin{array}{l}\text { Standard } \\
\text { size } \mathbf{m m}\end{array}$ & $\begin{array}{l}\text { Wings } \\
\text { size } \mathbf{m m}\end{array}$ & $\begin{array}{c}\text { Keel size } \\
\text { mm }\end{array}$ & & $\begin{array}{c}\text { Length of } \\
\text { stamen } \\
\text { tube mm }\end{array}$ & $\begin{array}{l}\text { Height of } \\
\text { stamen } \\
\text { tube mm }\end{array}$ & $\begin{array}{c}\text { Length of } \\
\text { cohesion } \\
\text { distance } \\
\text { mm }\end{array}$ & $\begin{array}{c}\text { f Length } \\
\text { of free } \\
\text { stamen } \\
\text { mm }\end{array}$ & Tip shape & \\
\hline T. apollinea & Pink & 11.5 & $10.5 \times 11.5$ & $5 \times 11$ & $3 \times 7$ & Club & 7 & 1.5 & 5 & 6.25 & Quardrate & $\begin{array}{l}\text { Vertical on } \\
\text { tube }\end{array}$ \\
\hline T. desertorum & Deep pink & 9.5 & $10 \times 9.5$ & $4.5 \times 9.5$ & $3.25 \times 6.75$ & Club & 7.5 & 2 & 5 & 5.75 & Quardrate & $\begin{array}{l}\text { Toward } \\
\text { anther }\end{array}$ \\
\hline T. leptostachya & Pink & 8 & $8 \times 8$ & $2.5 \times 7$ & $2.25 \times 5.75$ & Club & 5.5 & 1.25 & 4 & 5 & Quardrate & $\begin{array}{l}\text { Vertical on } \\
\text { tube }\end{array}$ \\
\hline T. nubica & $\begin{array}{l}\text { Pale } \\
\text { pink }\end{array}$ & 13 & $13 \times 14$ & $6 \times 14.5$ & $4.5 \times 11.5$ & Boat & 11 & 3 & 6.75 & 10 & Triangle & $\begin{array}{l}\text { Vertical on } \\
\text { tube }\end{array}$ \\
\hline T. pubescens & Pink & 10.25 & $8 \times 10.5$ & $4 \times 9.75$ & $3 \times 7.25$ & Club & 6.5 & 1.5 & 4 & 5.5 & Quardrate & $\begin{array}{l}\text { Toward } \\
\text { anther }\end{array}$ \\
\hline T. pumila & $\begin{array}{l}\text { Pale } \\
\text { pink }\end{array}$ & 7.5 & $5 \times 7.5$ & $2 \times 7$ & $2.75 \times 7$ & Kidney & 7 & 1 & 5 & 5.5 & Triangle & $\begin{array}{l}\text { Vertical on } \\
\text { tube }\end{array}$ \\
\hline T. purpurea & $\begin{array}{l}\text { Pale } \\
\text { pink }\end{array}$ & 10.5 & $9 \times 10.5$ & $4 \times 10.5$ & $3.25 \times 6$ & Boat & 8.75 & 1.5 & 6 & 8 & Triangle & $\begin{array}{l}\text { Vertical on } \\
\text { tube }\end{array}$ \\
\hline T. quartiniana & $\begin{array}{l}\text { Pale } \\
\text { pink }\end{array}$ & 7 & $7.5 \times 7$ & $3 \times 8$ & $2.75 \times 7$ & Boat & 7.25 & 1.75 & 4.5 & 6 & Triangle & $\begin{array}{l}\text { Vertical on } \\
\text { tube }\end{array}$ \\
\hline T. uniflora & Pale pink & 8.25 & $8 \times 8.25$ & $3 \times 7.5$ & $3 \times 7.5$ & Boat & 8 & 1.25 & 5.5 & 6.5 & Triangle & $\begin{array}{l}\text { Vertical on } \\
\text { tube }\end{array}$ \\
\hline
\end{tabular}

Table 5. Gynoecium and fruits characters of the studied Tephrosia species.

\begin{tabular}{|c|c|c|c|c|c|c|c|c|c|c|}
\hline \multirow[b]{2}{*}{ Species } & \multicolumn{3}{|c|}{ Gynoecium } & \multicolumn{7}{|c|}{ Fruits } \\
\hline & $\begin{array}{c}\text { Carpel } \\
\text { length } \\
\text { mm }\end{array}$ & $\begin{array}{c}\text { Ovary size } \\
\text { Mm }\end{array}$ & $\begin{array}{c}\text { Hairs on } \\
\text { ovary }\end{array}$ & Color & Shape & Dehiscence & $\begin{array}{l}\text { Numbers of } \\
\text { seeds }\end{array}$ & $\begin{array}{l}\text { Size } \\
\text { mm }\end{array}$ & $\begin{array}{c}\text { Trace of } \\
\text { seed }\end{array}$ & $\begin{array}{l}\text { Presence of } \\
\text { membrane }\end{array}$ \\
\hline T. apollinea & 8 & $0.75 \times 4$ & Short & $\begin{array}{l}\text { Yellowish } \\
\text { brown }\end{array}$ & Linear & Dehiscent & $7-5$ & $0.35 \times 4.3$ & Present & Present \\
\hline T. desertorum & 8.25 & $0.75 \times 4.5$ & Short & Brown & Linear & Dehiscent & $8-6$ & $0.3 \times 4$ & Present & Present \\
\hline T. leptostachya & 6.5 & $0.5 \times 3.25$ & Short & Yellow & Linear & Dehiscent & $8-7$ & $0.25 \times 2.8$ & Present & Present \\
\hline T. nubica & 12 & $1.75 \times 6$ & Long & Cream & Ovoid & Not dehiscent & 1 & $0.6 \times 1.2$ & Absent & Absent \\
\hline T. pubescens & 8.5 & $0.75 \times 4.5$ & Short & $\begin{array}{l}\text { Yellowish } \\
\text { brown }\end{array}$ & Linear & Dehiscent & $8-7$ & $0.3 \times 3.8$ & Present & Present \\
\hline T. pumila & 7.75 & $1 \times 4.25$ & Medium & Dark brown & Linear & Dehiscent & $13-9$ & $0.3 \times 3.8$ & Absent & Absent \\
\hline T. purpurea & 10.25 & $0.75 \times 5.25$ & Medium & Dark brown & Linear & Dehiscent & $7-5$ & $0.35 \times 3.25$ & Absent & Absent \\
\hline T. quartiniana & 7.75 & $0.75 \times 4$ & Medium & Yellow & Linear & Dehiscent & $10-7$ & $0.4 \times 2.75$ & Absent & Present \\
\hline T. uniflora & 9.25 & $0.75 \times 4.75$ & Short & Yellow & Linear & Dehiscent & $11-10$ & $0.3 \times 5.3$ & Absent & Absent \\
\hline
\end{tabular}

All measures are given as a mean. 


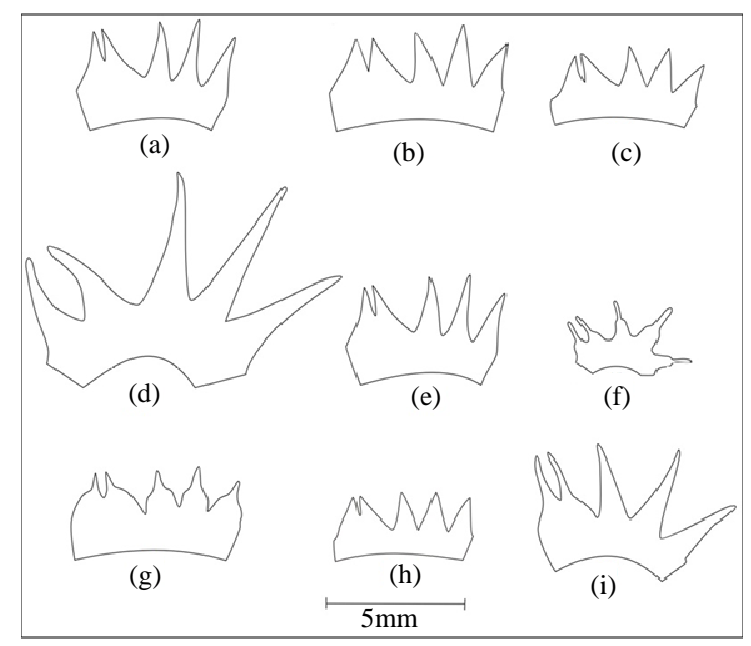

Figure 2. Schematic diagram showing sepals shape of studied Tephrosia species: (a) T. apollinea; (b) T. desertorum; (c) T. leptostachya; (d) T. nubica; (e) T. pubescens; (f) T. pumila; (g) T. purpurea; (h) T. quartiniana; (i) T. uniflora.

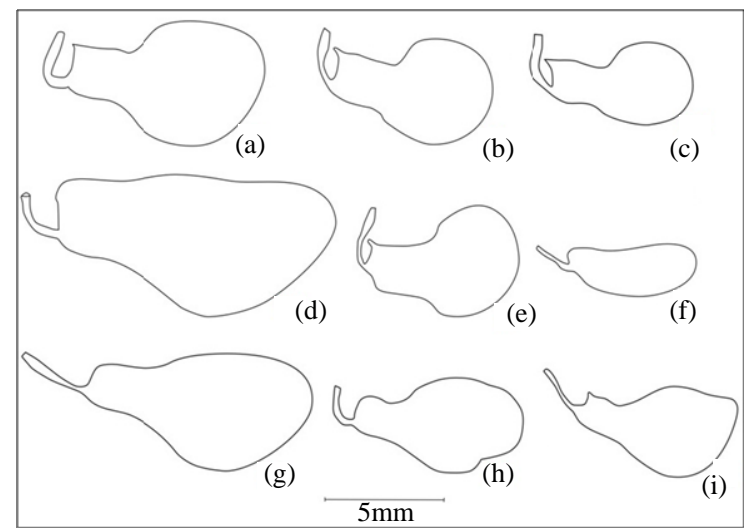

Figure 3. Schematic diagram showing wings shape of corolla of the studied Tephrosia species: (a) T. apollinea; (b) T. desertorum; (c) T. leptostachya; (d) T. nubica; (e) T. pubescens; (f) T. pumila; (g) T. purpurea; (h) T. quartiniana; (i) T. uniflora.

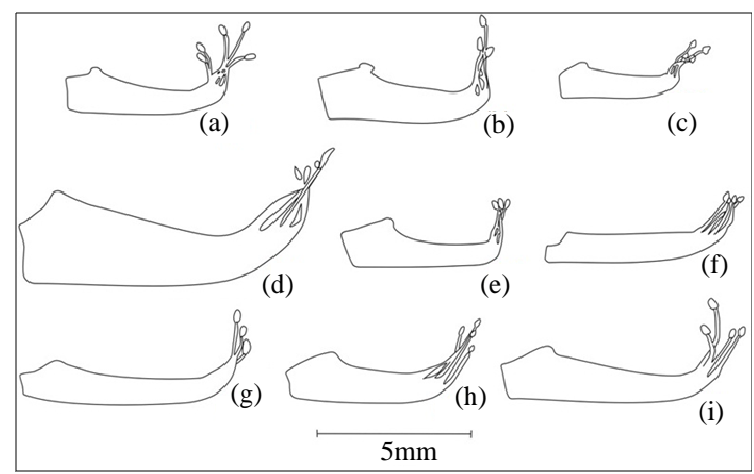

Figure 4. Schematic diagram showing side view of stemnal tube shape of androecium of the studied Tephrosia species: (a) T. apollinea; (b) T. desertorum; (c) T. leptostachya; (d) T. nubica; (e) T. pubescens; (f) T. pumila; (g) T. purpurea; (h) T. quartiniana; (i) T. uniflora. size $0.6 \times 1.2 \mathrm{~cm}$, covered with creamy long hairs, not dehiscent at maturity.

\subsection{Systematic Treatment}

1. Pod ovoid, one seeded, covered with creamy long hairs, not dehiscent at maturity, upper 2 teeth of calyx cleft ................................................. T. nubica

+Pod linear, many seeded, covered with short to medium white hairs, dehiscent at maturity, upper 2 teeth of calyx dentate .......................................... 2

2. Inflorescence axillary................................. 3

+Inflorescence pseudoracemes borne terminal (opposite a leaf)...................................................4

++Inflorescence pseudoracemes borne terminal (opposite a leaf) and/or axillary..... T. purpurea

3. Leaflets obvate pubescent on both surfaces.................................... T. quartinian +Leaflets oblanceolates glabrous on the upper surface ....................................... T. uniflora

4. Day flowering with kidney wings of

corolla............................................ T. pumila

+Flowering evening with club wings of corolla ........5

5. Perennial, leaflets obvate or oblanceolates, with emarginate or apiculate apex. . .6

+Annual, leaflets obvate with obcordate apex ........7

6. Stem erect, leaflets obovate, with emarginate apex, long pinnose hairs, tip of staiminal tube vertical to the tube. T. apollina

+Stem ascending, leaflets oblanceolate, with apiculate apex. Pubescent hairs, staminal tube toward stamen ....................................T. pubescencs

7. Stem asending, pubescent hairs, staminal tube toward anther T. desortium

+Stem prostate, tip of staiminal tube vertical to the tube. T. leptostachya

\section{REFERENCES}

[1] J. C. Willis, "Dictionary of Flowering Plant and Ferns," University Press, Cambridge, 1973, p. 1135.

[2] W. Zhi and L. Pedley, “Tephrosia Pers,” Flora of China, Vol. 10, 2010, pp. 190-193.

[3] S. Collenette, "An Illustrated Guide to the Flowers of Saudi Arabia," Scorpion publishing Ltd., London, 1985.

[4] A. M. Migahid, "Flora of Saudi Arabia," 2nd Edition, King Saud University Libraries Publication, Riyadh, Vol. 2, 1989.

[5] S. Collenette, "Wild Flowers of Saudi Arabia," National Commission for Wildlife Conservation and Development (NCWCD), Riyadh, 1999.

[6] S. A. Chaudhary and A. A. Al-Jawaid, "Vegetation of the Kingdom of Saudi Arabia,” Ministry of Agriculture and Water, Riyadh, 1999.

[7] S. A. Chaudhary, "Flora of the Kingdom of Saudi Arabia 
(Vascular Plants),” National Agriculture and Water Research Center, National Herbarium, Ministry of Agriculture and Water, Riyadh, 2001.

[8] D. R. Kaplan “The Science of Plant Morphology: Definition, History, and Role in Modern Biology,” American Journal of Botany, Vol. 88, No. 10, 2001, pp. 1711-1741. doi: $10.2307 / 3558347$

[9] L. Boulos, “Flora of Egypt,” Al-Hadara Publishing, Cairo, 1999.

[10] A. E. Radford, W. C. Dickison, J. R. Massey and C. R.
Bell, “Vascular Plant Systematics," Harper and Row, New York, 1974.

[11] V. Tackholm, "Students Flora of Egypt," 2nd Edition, Cairo University, Giza, 1974.

[12] S. C. Tucker, "Floral Development in Legumes," Plant Physiology, Vol. 131, No. 3, 2003, pp. 911-926. doi:10.1104/pp.102.017459

[13] H. A. Hosni and Z. A. R. El-Karemy, "Systematic Revision of Leguminosae in Egypt. 1. Tephrosia Pers.," Sendtnera, Vol. 1, 1993, pp. 245-257. 